\title{
Residual cholesteatoma of the mastoid cavity presenting with radiologic signal of a cholesterol granuloma: A case report
}

\author{
Marilyn Isabel $^{1}$, Jean Chénard ${ }^{1}$, Margaret Aron*2 \\ ${ }^{1}$ Radiology Department of the Centre Hospitalier Universitaire de Sherbrooke, Université de Sherbrooke, Canada \\ ${ }^{2}$ Otolarnygology-Head and Neck Surgery Department of the Centre Hospitalier Universitaire de Sherbrooke, Université de \\ Sherbrooke, Canada
}

Received: May 14, 2018

Accepted: September 2, 2018

Online Published: September 4, 2018

DOI: $10.5430 /$ css.v4n2p21

URL: https://doi.org/10.5430/css.v4n2p21

\begin{abstract}
Introduction: Cholesteatoma and cholesterol granuloma can both occur in the temporal bone. Their distinct magnetic resonance imaging (MRI) signals are generally used to help differentiate one from the other to plan appropriate treatment, which differs based on the pathology.

Case presentation: We present a patient with recent-onset facial paralysis whose mastoid cavity was filled with tissue radiologically resembling cholesterol granuloma but that turned out to be cholesteatoma upon surgical exploration. Complete disease extirpation was thus completed rather than simple marsupialization, the latter of which would have been sufficient in the case of cholesterol granuloma.

Discussion: Cholesteatoma, although usually hypointense on T1-weighted imaging (T1WI) MRI, hyperintense on T2-weighted imaging (T2WI) MRI and showing diffusion restriction on diffusion-weighted imaging can occasionally present with an atypical MRI signal, for example, one suggestive of cholesterol granuloma.

Conclusions: The clinician should keep in mind the possible diagnosis of cholesteatoma despite an atypical MRI signal in the temporal bone.
\end{abstract}

Key Words: Atypical cholesteatoma, White cholesteatoma, White epidermoid, Cholesterol granuloma, Mastoid

\section{INTRODUCTION}

Cholesteatomas are common benign lesions composed of a matrix of epithelial lining filled with keratin debris. They arise principally in the middle ear and mastoid, may arise in any other part of the temporal bone and can be either congenital (15\%) or acquired (85\%). In the skull base, cholesterol granuloma is the main differential diagnosis.

On Non-enhanced computed tomography (NECT) imaging, cholesteatoma is usually hypo- to hyperdense and cholesterol granuloma is spontaneously hyperdense making CT alone insufficient in distinguishing these two entities. On MRI, however, their differing and distinct signal characteristics facilitate the differentiation of one from the other. Cholesteatoma, or epidermoid cyst, is hypo or isointense on T1-weighted imaging (T1WI) magnetic resonance imaging (MRI), hyperintense on T2-weighted imaging (T2WI) MRI, does not enhance with contrast and shows frank diffusion

\footnotetext{
*Correspondence: Margaret Aron; Email: maggiearon@gmail.com; Address: Service d'ORL; Faculté de Médecine de l’Université de Sherbrooke, 580, rue Bowen Sud, Sherbrooke, Qc J1G 2E8, Canada.
} 
restriction on diffusion weighted imaging (DWI). ${ }^{[1]}$ Choles- for cure, thus the pre-operative diagnosis, highly dependent terol granuloma, although also hyperintense on T2WI and devoid of contrast enhancement, is characteristically hyperintense on T1WI and does not show diffusion restriction on DWI. ${ }^{[1]}$

Clinically, it is important to differentiate between these two diagnoses in order to guide treatment: whereas symptomatic cholesterol granulomas can simply be marsupialized and drained, cholesteatomas require complete disease extirpation on imaging characteristics, can significantly alter the planned extent of surgery.

\section{CASe PResentation}

A 53-year-old patient with a history of chronic ear disease presented to the Otolaryngology clinic with right-sided otalgia and recent onset facial paralysis.
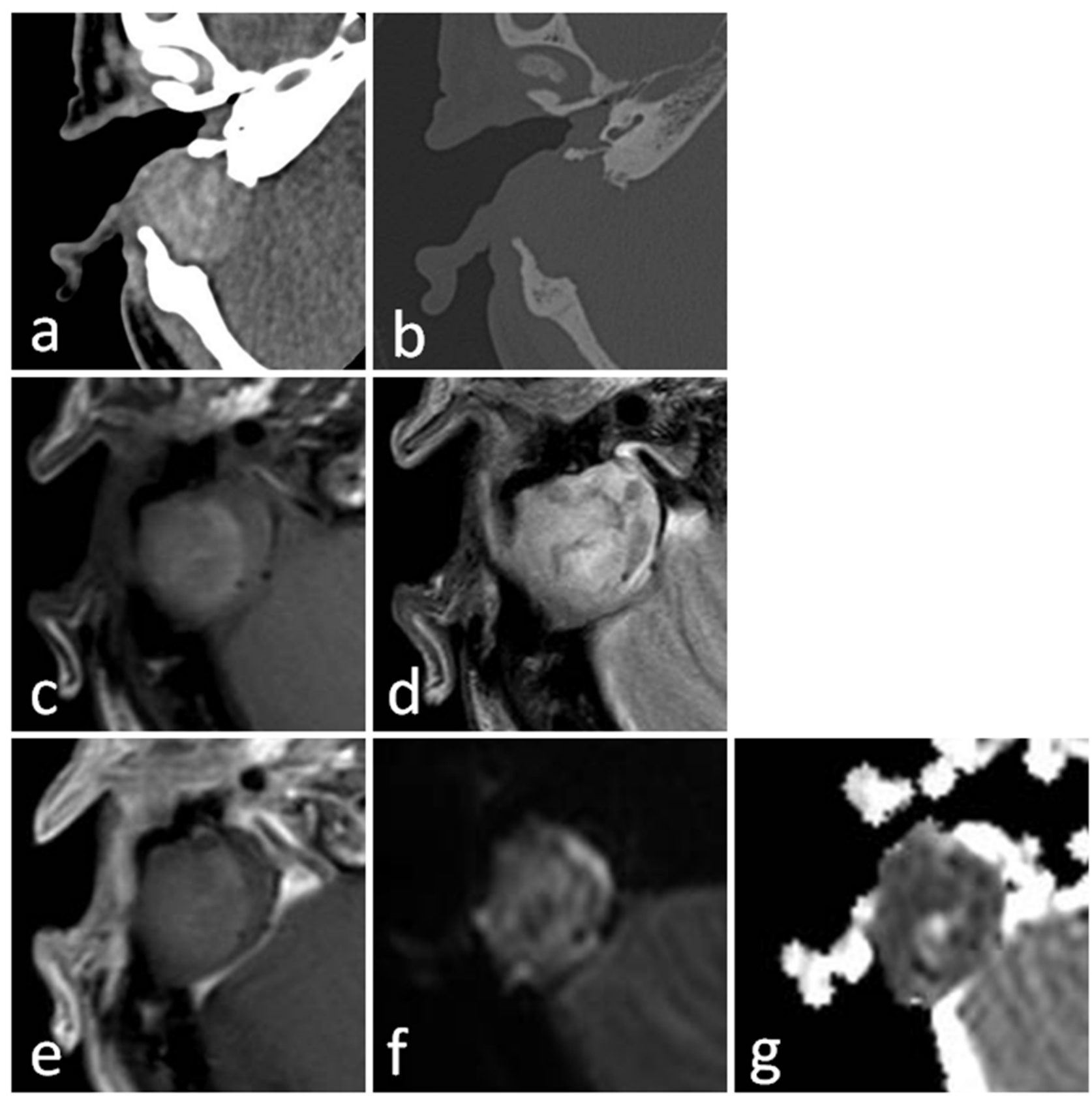

Figure 1. NECT and 3T-MRI axial acquisitions centered on the right mastoid cavity. The soft-tissue (a) and bone (b) windows show a spontaneously hyperdense lesion eroding the right mastoid cavity. The non-enhanced T1W-spin-echo (c) and T2W-turbo-spin-echoSE (d), gadolinium-enhanced T1W-SE (e), DWI b1,000 (f) and DWI ADC-map (g) 
He had previously undergone two surgeries on his right ear. The most recent surgery, 24 years prior, was a revision modified radical tympano-mastoidectomy with dissection of extensive residual cholesteatoma from the mastoid cavity, including off of a largely exposed vertical (mastoid) facial nerve.

At the time of presentation to clinic the patient was not toxic and showed no signs of meningeal irritation. His mastoid cavity was dry other than a small zone of granulation tissue posteriorly. He had a House-Brackmann grade 5 facial paralysis on the affected side.

NECT imaging revealed a round expansile lesion of the right temporal bone eroding the posterior wall of the previously drilled mastoid cavity. The lesion was heterogeneous and hyperdense relative to brain (see Figure $1 \mathrm{a}-\mathrm{b}$ ). There was a thin and partially calcified rim around the lesion but no central calcification. There was erosion of the osseous plate of

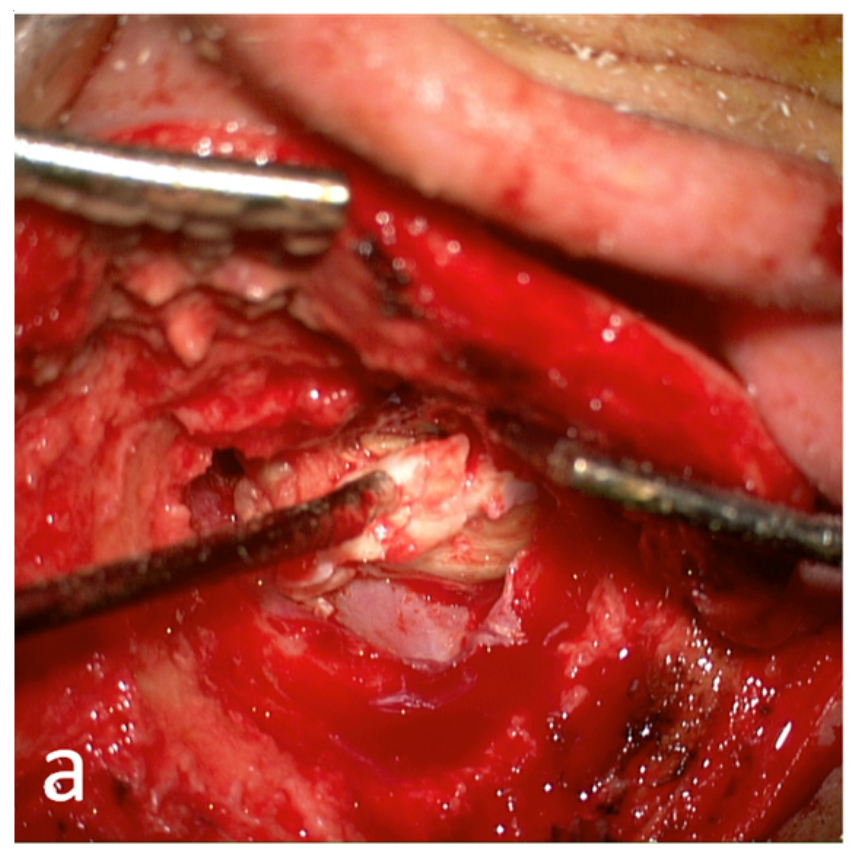

the right sigmoid sinus and jugular bulb, without thrombosis. The bony canal of the mastoid portion of the facial nerve was also eroded.

MRI showed a heterogenous lesion in the right mastoid cavity measuring $26 \mathrm{~mm} \times 23 \mathrm{~mm} \times 28 \mathrm{~mm}$ and containing internal striations. The lesion was spontaneously hyperintense on T1WI and T2WI, and showed no enhancement after gadolinium injection (see Figure $1 \mathrm{c}-\mathrm{e}$ ). On DWI sequences and apparent diffusion coefficient (ADC) map, the diffusion was heterogeneous showing only slight peripheral diffusion restriction (see Figure $1 \mathrm{f}-\mathrm{g}$ ). The osseous wall of the mastoid portion of the facial nerve was absent, and the nerve itself was difficult to isolate from the lesion.

Surgical exploration revealed keratin debris in concentric layers filling and expanding the mastoid cavity (see Figure 2). Pathologic evaluation confirmed the surgical findings of cholesteatoma.

Figure 2. Peri-operative photos showing the classic pearly-white multi-layered appearance of the cholesteatoma

\section{Discussion}

Our patient presented with a right mastoid, pathologyconfirmed cholesteatoma, but with an MRI signature more typical of a cholesterol granuloma. These "white epidermoids" are a rare entity, with only a few cases described in the literature, all of which were intracranial lesions (epidermoid cysts). ${ }^{[2,4-6]}$ A literature review did not retrieve any case of "white cholesteatoma" of the temporal bone or mastoid cavity.

In 2015, Dinh et al. reported a case of pathology-proven Published by Sciedu Press

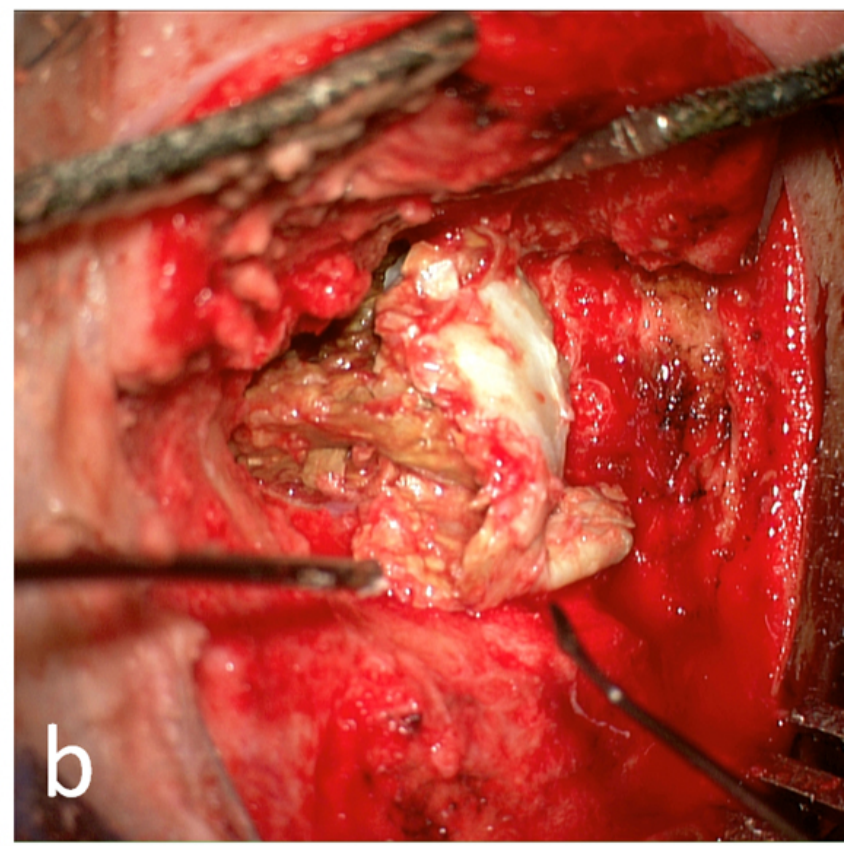

cholesterol granuloma mistaken for a cholesteatoma based on CT and MRI data, ${ }^{[3]}$ which was iso-intense on T1WI, hyperintense on T2WI and slightly enhancing peripherally after gadolinium infusion. This stresses the multiple radiologic signatures of these two entities and the associated diagnostic challenge.

Like intracranial epidermoid cysts, cholesteatomas are made up of keratin, protein, debris and cholesterol, with the proportion of these components varying from one particular cholesteatoma to another. It has ben postulated that, at least for epidermoid cysts, the cholesterol and calcium content 
could change the T1WI appearance of the lesion. ${ }^{[4]}$ Three papers reported cases of intracranial epidermoid cysts that were hyperintense on both T1WI and T2WI sequences and attributed this unusual appearance to high viscosity and protein content. ${ }^{[5-7]}$ Being so closely related to epidermoid cyst that many authors consider them to be the same entity, the imaging signature of cholesteatomas is thus certainly influenced in the same way by its own composition and viscosity.

Clinically, it is important to keep this imaging variability in mind and consider the differential to guide treatment and obtain appropriate pre-operative consent. Whereas symptomatic cholesterol granulomas can simply be marsupialized and drained, cholesteatomas require complete disease extirpation for cure. In this case that dictated the difference between dissecting disease off of an already damaged and dehiscent facial nerve and dura (with their inherent complication risks) versus simple marsupialisation and avoidance of these structures.

\section{Conclusions}

Clinically, it is important to keep in mind the possibility of a "white epidermoid" in the temporal bone, including the mastoid cavity. This cholesteatoma can have a radiologic signal more suggestive of cholesterol granuloma but requires complete extirpation for cure and thus should be considered pre-operatively in order to appropriately guide consent, surgical planning and definitive surgical treatment.

\section{Consent}

Consent from the patient was obtained to publish his case without any nominal or identifying data.

\section{CONFLICTS OF INTEREST Disclosure}

The authors declare they have no conflicts of interest.

\section{REFERENCES}

[1] Barath K, Huber AM, Stämpfli P, et al. Neuroradiology of cholesteatomas. Am J Neuroradiol. 2011; 32: 221-29. PMid: 20360335. https://doi.org/10.3174/ajnr.A2052

[2] Law EKC, Lee RKL, Ng AWH, et al. Atypical intracranial epidermoid cysts : rare anomalies with unique radiological features. Case Rep Radiol. 2015; 2015: 528632.

[3] Dinh CT, Goncalves S, Bhatia R, et al. Atypical radiographic features of skull base cholesterol granuloma. Eur Arch Otorhinolaryngol. 2016; 273: 1425-31. PMid: 26164292. https ://doi .org/10.100 7/s00405-015-3705-7

[4] Gualdi GF, Di Biasi C, Trasimeni G, et al. Unusual MR and CT appearance of an epidermoid tumor. Am J Neuroradiol. 1991; 12: 717-79.

[5] Takeshita M, Kubo O, Hiyama H, et al. Magnetic resonance inaging and quantitative analysis of contents of epidermoid and dermoid cysts. Neurol Med Chir. 1994; 34: 436-439. https://doi.org/10.217 $6 / \mathrm{nmc} .34 .436$

[6] Timmer FA, Sluzewski M, Treskes M, et al. Chemical analysis of an epidermoid cyst with unusual CT and MR characteristics. Am J Neuroradiol. 1998; 19: 1111-1112. PMid: 9672020.

[7] Ochi M, Hayashi K, Hayashi T, et al. Unusual CT and MR appearance of an epidermoid tumor of the cerebellopontine angle. Am J Neuroradiol. 1998; 19: 1113-1115. PMid: 9672021. 